\title{
5. Über den Einfluß von Wänden auf die Bewegung einer Kugel in einer reibenden Flüssigkeit ${ }^{1}$ ); von Rudolf Ladenburg.
}

Fällt eine Kugel unter dem EinfluB der Schwere in einer reibenden Flüssigkeit, so ist nach einer Berechnung von G. G. Stokes ${ }^{2}$ ) die Kugelgeschwindigkeit nur abhängig von dem Auftrieb und dem Radius der Kugel und der inneren Reibung der Flüssigkeit, vorausgesetzt, daß diese Geschwindigkeit genügend klein und die äuBere Reibung zwischen Kugel und Flüssigkeit unendlich groB ist. Praktisch kann eine solche Beziehung jedoch nur als erfüllt angesehen werden, wenn die Bewegung der Kugel weder durch benachbarte andere Kugeln gestört wird noch durch Wände, die die Flüssigkeit begrenzen. So zeigten Experimente ${ }^{3}$ ) in zylindrischen, beiderseitig geschlossenen GefäBen noch einen merklichen hemmenden EinfluB der Wände, als die Dimensionen der Gefäße die der Kugel um fast das 100 fache übertrafen, und es schien mir deshalb wichtig, den Einfluß solcher Begrenzungen auch theoretisch zu berechnen.

Ich möchte auch an dieser Stelle Hrn. Prof. Korn meinen aufrichtigen Dank sagen, der mir den Weg zur Ausrechnung zeigte und mich bei der Ausführung in liebenswürdigster Weise unterstützte.

Man kann den Einfluß beliebiger Flächen auf die Bewegung eines Körpers in einer reibenden Flüssigkeit nach H. A. Lorentz ${ }^{4}$ ) durch Superposition der Zustände bestimmen,

1) Die Resultate vorliegender Arbeit sind zum Teil schon in der Münchner Dissertation des Verf. (Leipzig 1906) enthalten und bilden eine theoretische Ergänzung zu der Mitteilung des Verf. „Über die innere Reibung zäher Flüssigkeiten und ihre Abhängigkeit vom Druck" Ann. d. Phys. 23. p. 287. 1907.

2) G. G. Stokea, Cambr. Phil. Trans. 8. p. 287; 9. p. 8. 1850.

3) R. Ladenburg, l. c.

4) H. A. Lorentz, Zittingsveri. Akad. van Wet. 5. p. 168. 1896; neu bearb.: "Abhandl. über theor. Phys." 1'. p. 23 ff. 1906 (Teubners Verlag). Dieselbe Methode findet sich bereits bei G. G. Stokes, Cambr. Phil. Soc. 8. 1843. 
die durch Reflexion („Zurückwerfung") der ursprünglichen Bewegung an den Flächen und an dem Körper selbst entstehen. Sind die Dimensionen des Körpers unendlich klein und geht die Bewegung rom Körper selbst aus, so genügt') die Kenntnis der ersten Reflexion von jenen Flächen; ist der Körper z. B. eine Kugel, so kann $\operatorname{man}^{1}$ ) aus der Geschwindigkeit, die infolge der ursprünglichen Bewegung und jener ersten Reflexion bei Abwesenheit der unendlich kleinen Kugel an ihrem Mittelpunkte herrschen würde, den Widerstand berechnen, den die Kugel bei ihrer Bewegung erfährt.

Bei der Bewegung einer Kugel in einer geschlossenen Röhre wäre also die Reflexion der für den unendlichen Raum geltenden Bewegung von einem begrenzten Zylinder zu berechnen; da ich hierbei auf unüberwindliche Schwierigkeiten stieb, habe ich mich auf die Berechnung und Superposition der Geschwindigkeiten beschränken müssen, die durch Reflexion von einem unendlich langen Zylinder und von zwei unendlich ausgedehnten Ebenen entstehen, deren Abstand gleich der Röhrenlänge ist. Diese vereinfachenden Annahmen scheinen mir aber bereits eine beträchtliche Annäherung an die wahren Versuchsbedingungen zu liefern, da die Geschwindigkeiten, die die unendlich kleine Kugel hervorruft, auch in endlicher Entfernung unendlich klein ') sind.

Die hydrodynamischen Grundgleichungen der stationären Bewegung einer reibenden Flüssigkeit lauten ${ }^{2}$ ), wenn man die Glieder vernachlässigt, die Quadrate oder Produkte von Geschwindigkeiten enthalten, und wenn keine äußeren Kräfte auf die Flüssigkeit wirken:

$$
\begin{gathered}
k \Delta u=\frac{\partial p}{\partial x}, \quad k \Delta v=\frac{\partial p}{\partial y}, \quad k \Delta w=\frac{\partial p}{\partial z}, \\
\frac{\partial u}{\partial x}+\frac{\partial v}{\partial y}+\frac{\partial w}{\partial z}=0,
\end{gathered}
$$

wobei $u, v, w$ die Geschwindigkeitskomponenten der Flüssigkeitsteile in Richtung der Koordinatenachsen $X, Y, Z, k$ den Reibungskoeffizienten und $p$ den Druck bedeuten und die Flüssigkeit

1) H. A. Lorentz, l. c. p. 30 .

2) Vgl, z. B. G. Kirchh off, Vorl. über Mechanik, 4. Aufl. p. 378 ff. 1897, oder H. A. Lorentz, l. c. p. 30. 
als unbegrenzt vorausgesetzt ist. Bewegt sich in dieser Flüssigkeit eine Kugel vom Radius $R$ in Richtung der negativen $Z$-Achse mit der gleichförmigen Geschwindigkeit $a$, so genügen den Differentialgleichungen (1) und (2), wenn der Mittelpunkt der Kugel Koordinatenanfangspunkt und die äuBere Reibung zwischen Kugel und Flüssigkeit unendlich grob ist, die Funktionen $\left.{ }^{1}\right)$ :

$$
\left\{\begin{aligned}
u_{1} & =\frac{3}{4} a R^{3} \frac{x z}{E^{5}}-\frac{3}{4} a R \frac{x z}{E^{3}}, \\
v_{1} & =\frac{3}{4} a R^{3} \frac{y z}{E^{5}}-\frac{3}{4} a R \frac{y z}{E^{3}}, \\
w_{1} & =\frac{a R^{3}}{4}\left(\frac{3 z^{2}}{E^{5}}-\frac{1}{E^{3}}\right)-\frac{3}{4} a R\left(\frac{x^{2}}{E^{8}}+\frac{1}{E}\right), \\
p_{1} & =-\frac{3}{2} k R a \frac{z}{E^{3}}+\text { konst. }
\end{aligned}\right.
$$

wobei

gesetzt ist.

$$
E^{2}=x^{2}+y^{2}+z^{2}
$$

Um nun zunächst die Reflexion dieser Bewegung von einem unendlich langen Zylinder $z u$ berechnen, dessen Achse mit der $Z$-Achse zusammenfalle und dessen Querschnittsradius $\varrho$ sei, führe ich in der $X Y$-Ebene die Polarkoordinaten $r$ und $\rightarrow$ ein und setze

$$
x=r \cos \vartheta, \quad y=r \sin \vartheta \quad \text { und } \quad \frac{d r}{d t}=s .
$$

Berücksichtige ich, daB in diesem Falle $d \vartheta / d t$ offenbar Null ist und setze noch zur Abkürzung für eine beliebige Funktion $\varphi$

so wird

$$
\frac{\partial^{2} \varphi}{\partial z^{2}}+\frac{\partial^{2} \varphi}{\partial r^{2}}+\frac{1}{r} \frac{\partial \varphi}{\partial r}=D \varphi,
$$

$$
\Delta \varphi=D \varphi+\frac{1}{r^{2}} \frac{\partial^{2} \varphi}{\partial \vartheta^{3}}
$$

und durch Vernachlässigung der Glieder mit $R^{3}$ in den Gleichungen (3) gehen die Gleichungen (1), (2) und (3) über in

$$
\left\{\begin{array}{r}
D s-r_{r^{2}}^{s}=\frac{1}{k} \frac{\partial p}{\partial r}, \\
D w=\frac{1}{k} \frac{\partial p}{\partial z}, \\
\frac{\partial s}{\partial r}+\frac{s}{r}+\frac{\partial w}{\partial z}=0,
\end{array}\right.
$$

1) G. Kirchhoff, l. c., oder H. A. Lorentz, l. c. Aunalen der Physik. IV. Folgo. 23, 


$$
\left\{\begin{array}{c}
s_{1}=-\frac{3}{4} a R \frac{r z}{E^{3}}, \\
w_{1}=-\frac{3}{4} a R\left(\frac{z^{2}}{E^{3}}+\frac{1}{E}\right), \\
p_{1}=-\frac{3}{2} k R a \frac{z}{E^{3}}+\text { konst. }, \\
E^{2}=r^{2}+z^{2} .
\end{array}\right.
$$

Es handelt sich nun darum, neue Lösungen - im folgenden durch den Index 2 charakterisiert - der Differentialgleichungen (1a) und (2a) zu finden, die den Bedingungen genügen:

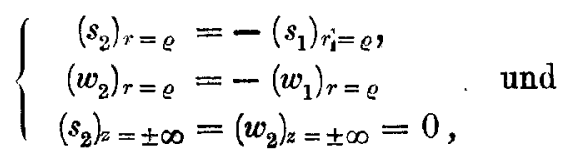

da dann offenbar der Zustand 2 die gesuchte, durch Reflexion an der Zylinderwand entstandene, Bewegung darstellt.

$\mathrm{Zu}$ diesem $\mathrm{Zwecke}$ führe ich die Besselschen oder Zylinderfunktionen erster und zweiter Art $0^{\text {ter }}$ Ordnung, $J(\Theta)$ und $K(\Theta)$, ein, die partielle Lösungen der Besselschen Differential. gleichung

$$
\frac{d^{2} z}{d \Theta^{2}}+\frac{1}{\Theta} \frac{d z}{d \Theta}+z=0
$$

sind und nach Hein $\mathrm{e}^{1}$ ) darstellbar in der Form:

$$
J(\Theta)=1-\frac{\Theta^{2}}{2^{2}}+\frac{\Theta^{4}}{2^{2} \cdot 4^{2}}-\frac{\Theta^{8}}{2^{2} \cdot 4^{2} \cdot 6^{2}}+\cdots
$$

$$
\left\{\begin{aligned}
K(\Theta)= & -J(\Theta)\left(\ln _{e} \frac{\Theta}{2}+C\right) \\
& -2\left[J_{2}(\Theta)-\frac{1}{2} J_{4}(\Theta)+\frac{1}{3} J_{6}(\Theta)-\ldots\right]
\end{aligned}\right.
$$

Hierbei sind $J_{2}, J_{4} \ldots$ die geraden Zylinderfunktionen erster Art höherer Ordnung und $C$, die sogenannte Gausssche ${ }^{2}$ ) Konstante, hat den Wert $^{3}$ )

$$
C=+0,5772157 \text {. }
$$

1) Heine, Theorie der Kugelfunktionen, 2. Aufl. 1. p. $184 \mathrm{ff}$. unt 2. p. 175.1878 .

2) K. F. Gauss, Ges. Werke III. p. 154, $C$ hat den Wert von $-\psi_{0}$.

3) Heine (l. c.) schreibt zwar $-0,5772 \ldots$, doch zeigt ein Vergleich mit der Originalliteratur (Gauss, l. c. und H. F. Weber, Borch. 
Einfluß von Wänden auf die Bewegung einer Kugel etc. 451

Nun ergibt sich aus den Gleichungen (1 a), daB

$$
D p=0
$$

ist; ist also $p_{2}$ der den Geschwindigkeiten $s_{3}$ und $w_{2}$ entsprechende Druck und $\lambda$ eine von Null verschiedene Zahl, so kann man für $p_{2}$ den Ansatz machen:

$$
p_{2}=\frac{2 k}{\pi} \int_{0}^{\infty} f(\lambda) \sin \lambda z J(i \lambda r) d \lambda,
$$

wobei die Funktion $f(\lambda)$ noch näher zu bestimmen ist. Dann läbt sich zeigen ${ }^{1}$ ), daB die Gleichungen (1 a) befriedigt werden durch die Funktionen:

$$
\left\{\begin{array}{l}
s_{2}=\frac{2}{\pi} \int_{0}^{\infty}\left[\frac{r}{2} f(\lambda) J(i \lambda r)+G(\lambda) J^{\prime}(i \lambda r)\right] \sin \lambda z d \lambda \\
\text { und } \\
w_{2}=\frac{2}{\pi} \int_{0}^{\infty}\left[\frac{i r}{2} f(\lambda) J^{\prime}(i \lambda r)+H(\lambda) J(i \lambda r)\right] \cos \lambda z d \lambda ;
\end{array}\right.
$$

hierin ist

$$
J^{\prime}(i \lambda r) \text { für } \frac{\partial J(i \lambda r)}{\partial(i \lambda r)}
$$

gesetzt und $G(\lambda)$ und $H(\lambda)$ sind zunächst willkürliche Funktionen. Da aber $s_{2}$ und $w_{2}$ den zwei Bedingungsgleichungen (4) und der Kontinuitätsgleichung (2a) zu genügen haben, werden die drei Funktionen $f(\lambda), G(\lambda)$ und $H(\lambda)$ eindeutig bestimmbar. Wie sich zeigen wird, genügt im vorliegenden Falle die Kenntnis von $H(\lambda)$, und aus jenen drei Gleichungen ergibt sich ${ }^{1}$, wenn man

$$
\frac{1}{E}=\frac{1}{\sqrt{x^{2}+y^{2}+z^{2}}}
$$

Journ. f. Math. 75. p. 85. 1878), daB $C$ positiv ist. Bei imaginären Werten des Argumentes ist nach Heine (l. c.) in der Darstellung für $K(\Theta)$ der Ausdruck $\frac{\pi}{2} i J(\Theta)$ zu addieren, so daB $i$ im Argumente de Logarithmus fortfällt.

1) Zur genaueren Durchführung der Rechnung vgl. die Dissertation deg Verf. p. 79-90. 
durch den Ausdruck ${ }^{\mathbf{l}}$ )

$$
\frac{2}{\pi} \int_{0}^{\infty} K(i \lambda r) \cos \lambda z d \lambda
$$

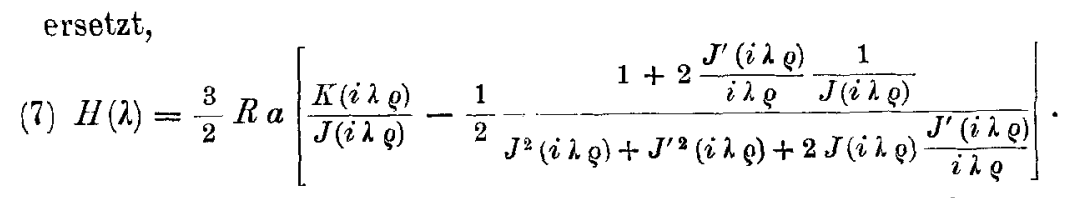

Wie in der Einleitung auseinandergesetzt, handelt es sich nun lediglich um die Berechnung der Werte, die $s_{2}$ und $w_{2}$ bei Abwesenheit der Kugel am Kugelmittelpunkte, d. h. am Koordinatenanfangspunkte besitzen: für $r=z=0$ folgt aus den Gleichungen (6)

$$
\begin{aligned}
\left(s_{2}\right) & =0, \\
\left(w_{2}\right) & =\frac{2}{\pi} \int_{0}^{\infty} H(\lambda) d \lambda=\frac{R}{\varrho} a \frac{3}{\pi} \int_{0}^{\infty} \varphi(i \lambda \omega) d(\varrho \lambda),
\end{aligned}
$$

wenn ich die Klammer auf der rechten Seite der Gleichung (7) abkürzend durch $\varphi(i \lambda \varrho)$ bezeichne.

Zur zahlenmäBigen Berechnung des Integrals

$$
\int_{0}^{\infty} \varphi(i \lambda \rho) d(\rho \lambda)
$$

habe ich in meiner Dissertation ${ }^{2}$ ) mit Benutzung der unendlichen Reihen (Gleichung $(5 b)$ und $(5 c)$ ) die Werte von

$$
J(i \lambda \varrho), \quad J^{\prime}(i \lambda \sigma), \frac{J^{\prime}(i \lambda \varrho)}{i \lambda \varrho} \text { und } K(i \lambda \varrho)
$$

für gewisse, geeignet scheinende Werte des Argumentes berechnet, aus ibnen jedesmal $\varphi(i \lambda \rho)$ zusammengesetzt und so das bestimmte Integral nach der Simpsonschen Regel ausgewertet. Bei der Zusammensetzung von $\varphi(i \lambda \varrho)$ ist jedoch ein Rechenfehler unterlaufen, so daB sich statt der in der Dissertation $^{3}$ ) gegebenen Zahlen der Wert von $\varphi(i \lambda \rho)$

1) Vgl. Heine, 1. c.

2) Dissertation des Verf. p. 124-126.

3) p. 126 . 
Einfluß von Ẅänden auf die Bewegung einer Kugel etc. 453

und damit wird

$$
\begin{aligned}
& \text { für } \lambda \varrho=\frac{1}{s} \text { zu } \quad 2,79 \\
& " \quad,=\frac{1}{4}, 2,00 \\
& " \quad,=\frac{1}{2}, 1,35 \\
& \text { " }=1 \quad, 0,67 \\
& " \quad "=2 \quad, \quad 0,21 \\
& " \quad,=4,0,014 \text { ergibt, }
\end{aligned}
$$

$$
\int_{0}^{\infty} \varphi(i \lambda \varrho) d(\varrho \lambda)=2,45
$$

und

$$
\left(w_{2}\right)=2,4 \frac{R}{\varrho} a ;
$$

d. h. unter dem Einfluß eines unendlich langen Zylinders vom Querschnittsradius $\varrho$ würden sich die Flüssigkeitsteile am Mittelpunkte der Kugel mit der Geschwindigkeit $2,4(R / \rho) a$ in der der Kugelgeschwindigkeit entgegengesetzten Richtung - die Kugelgeschwindigkeit war in Richtung der negativen Z-Achse vorausgesetzt (p. 449) - bewegen und die relative Geschwindigkeit zwischen Kugel und Flüssigkeit würde den Wert

annehmen.

$$
a\left(1+2,4 \frac{R}{\varrho}\right)
$$

Um ferner den Einfluß zu berechnen, den zwei einander parallele, unendlich ausgedehnte Ebenen auf die Bewegung einer Kugel zwischen ihnen ausüben, kann man die Methode verwenden, die H. A. Lorentz') zur Berechnung der ,Zurückwerfung" von einer senkrecht zur Kugelbewegung liegenden Ebene angegeben hat. Legt man die $X Y$-Ebene in jene reflektierende Ebene, bezeichnet mit $\alpha$ den momentanen Abstand der Kugel von jener Ebene, mit $E^{\prime}$ die Entfernung eines Punktes $(x, y, z)$ von dem-

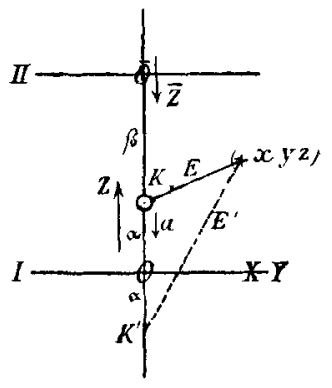
jenigen Punkte $K^{\prime}$ (vgl. Figur), der zum Kugelmittelpunkte $K$ bezüglich der Ebene symmetrisch liegt und läbt wie bisher die Richtung der Kugelgeschwindig-

1) H. A. Lorentz, ], c. 
keit mit der negativen $Z$-Richtung zusammenfallen, so erhält man für die allein in Betracht kommende $z$-Komponente der reflektierten Geschwindigkeit den Wert ${ }^{1}$ )

wobei

$$
w_{3}=\frac{3}{4} R a\left\{\frac{1}{E^{\prime}}+\frac{z^{2}+\alpha^{2}}{E^{\prime 3}}+\frac{6 \alpha z(z+\alpha)^{2}}{E^{\prime 5}}\right\},
$$

$$
E^{\prime 2}=x^{2}+y^{2}+(z+c)^{2}
$$

ist, und diese Bewegung würde am Mittelpunkte der Kugel eine der Kugelgeschwindigkeit entgegengesetzt gerichtete Geschwindigkeit der Flüssigkeitsteile von der Größe

hervorrufen.

$$
\underset{\substack{x=a \\ z=0}}{\left(w_{3}\right)^{y=0}}=\frac{9}{8} \frac{R}{\alpha} a
$$

Befindet sich nun auf der anderen Seite der Kugel im Abstande $\beta$ eine zweite Ebene (II), so würde diese offenbar am Kugelmittelpunkte zu einer Geschwindigkeit

$$
\left(w_{3}\right)^{\prime}=\frac{9}{8} \frac{R}{\beta} a,
$$

als Reflexion der ursprünglichen Bewegung, Veranlassung geben. ${ }^{2}$ ) Daneben ist aber offenbar auch die Zurückwerfung des Zustandes 3 von der Ebene II zu berücksichtigen. Um bei deren Berechnung die Lorentzsche Methode unmittelbar zu verwenden, habe ich die $X Y$-Ebene in die Ebene II verlegt und die Richtung der $Z$-Achse umgedreht $(\bar{Z}$-Achse). Dann erhalte ich am Kugelmittelpunkte die reflektierte Geschwindig. keit der Flüssigkeitsteile

$$
\left(\bar{w}_{4}\right)=\left(\frac{9}{8} \frac{1}{\alpha+\beta}+\frac{9}{8} \frac{\alpha \beta}{(\alpha+\beta)^{3}}\right) R a,
$$

welche diesmal mit der Richtung der Kugelgeschwindigkeit zusammenfällt, also den Widerstand, den die Kugel erfährt, verkleinert. Derselbe Wert würde sich aus Symmetriegränden für die Zurückwerfung des Zustandes $3^{\prime}$ von der Ebene $I$ ergeben. Reflektiert man weiter die Bewegung 4 an der Ebene I,

1) Vgl. H. A. Lorentz, l. c. p. 40.

2) $\mathrm{Da}$ sich die Kugel von dieser Ebene fortbewegt, ändert, wie die Rechnung von Lorentz zeigt, auch nichts an dem Vorzeichen der reflektierten Geschwindigkeit. 
Einfluß von Wänden auf die Bewegung einer Kugel etc. 455

so erhält man an der Stelle des Kugelmittelpunktes die Geschwindigkeit:

$$
\left(w_{5}\right)=\left(\frac{17}{18} \frac{1}{\alpha+\beta}+\frac{19}{27} \frac{\alpha \beta}{(\alpha+\beta)^{8}}\right) R a
$$

und denselben Wert offenbar für die entsprechende Reflexion an der Ebene II. Wie man sieht, werden die reflektierten Geschwindigkeiten allmählich kleiner, aber nicht von einer höheren Ordnung unendlich klein; doch genügt, wie sich zeigen wird, die durch die berechneten Reflexionen erreichte Genauigkeit.

Ich komme nun zur Bestimmung des Widerstandes. Bewegt sich die Kugel von einem Punkte

$$
z=(\alpha+\beta)-\frac{\alpha+\beta}{\gamma}=\frac{\gamma-1}{\gamma}(\alpha+\beta),
$$

wobei $\gamma>1$, bis zu einem Punkte

$$
z=\frac{\alpha+\beta}{\gamma}
$$

in Richtung der negativen Z-Achse, so ist der mittlere Wert der durch die einzelnen Reflexionen hervorgerufenen Geschwindigkeiten der Flüssigkeitsteile ${ }^{1}$ ), wenn $\operatorname{man} \alpha+\beta=h$ setzt, infolge der ersten beiden Reflexionen:

$$
\begin{gathered}
\frac{\gamma}{\gamma-2} \frac{1}{h} \int_{\frac{h}{\gamma}}^{\frac{\gamma-1}{\gamma} h}\left[\left(w_{3}\right)+\left(w_{3}\right)\right] d z=\frac{\gamma}{\gamma-2} \frac{1}{h} \int_{\frac{h}{\gamma}}^{\frac{\gamma-1}{\gamma} h} R a\left(\frac{1}{z}+\frac{1}{h-x}\right) d z \\
=a \frac{R}{h} \frac{9}{4} \frac{\gamma}{\gamma-2} \ln (\gamma-1)
\end{gathered}
$$

infolge der ersten und zweiten Reflexionen

$$
a \frac{R}{h}\left[\frac{9}{4} \frac{\gamma}{\gamma-2} \ln (\gamma-1)-\frac{27}{8}+\frac{9}{4} \frac{\gamma^{2}-\gamma+1}{3 \gamma^{2}}\right],
$$

und infolge der ersten, zweiten und dritten Reflexionen

$a \frac{R}{h}\left[\frac{9}{4} \frac{\gamma}{\gamma-2} \ln (\gamma-1)-\frac{27}{8}+\frac{9}{4} \frac{\gamma^{2}-\gamma+1}{3 \gamma^{2}}+\frac{70}{27}-\frac{38}{27} \frac{\gamma^{8}-\gamma+1}{3 \gamma^{2}}\right]$.

Um diese Beträge wird also die Geschwindigkeit der Kugel relativ zur Flüssigkeit vergröBert und dementsprechend auch der Widerstand, den die Kugel erfährt.

1) Bei dieser Berechnung ist die durch Variation des Widerstandes bewirkte geringe Änderung der Kugelgeschwindigkeit vernachlässigt. 
Um nun die Ergebnisse meiner Experimente ${ }^{1}$ ) mit dieser Berechnung $\mathrm{zu}$ vergleichen, ist hierin für $\gamma 3,5 \mathrm{zu}$ setzen, da die verwendeten Gefäße $24 \mathrm{~cm}$ lang waren und die Kugelgeschwindigkeit während der mittleren $10 \mathrm{~cm}$ beobachtet wurde. Aus obigen Formeln folgt dann für die Zusatzgeschwindigkeit $4,83 \frac{R}{h} a$ bez. $2,05 \frac{R}{h} a$ bez, $4,27 \frac{R}{h} a$,

so daB sich der za benutzende Wert durch geometrische Interpolation zu $3,3(R / h) a$ ergibt. Berücksichtigt man noch die nächsten Reflexionen, so erhält man einen nicht merklich verschiedenen Wert, so daB in der Tat die hier gegebene Berechnung der drei Reflexionen ausreicht. Werden nur je $2,4 \mathrm{~cm}$ an den Enden der GefäBe von der Beobachtung ausgeschlossen ${ }^{2}$ ), so ist $\gamma=10 \mathrm{zu}$ setzen, und man erhält als Zusatzgeschwindigkeit als Mittel den Wert 4,5(R/h)a.

Fasse ich also nun den Einfluß des unendlich langen Zylinders und der zwei Ebenen zusammen, d. h. superponiere ich direkt die betreffenden Zusatzgeschwindigkeiten, so erbält der Widerstand ${ }^{3}$ ), den die Kugel erleidet, statt

den Wert

$$
6 \pi k \cdot R a
$$

$$
6 \pi k R a\left(1+2,4 \frac{R}{\varrho}\right)\left(1+3,3 \frac{R}{h}\right),
$$

und dieser Ausdruck gleich

$$
\frac{4}{3} \pi R^{3}(s-\sigma)
$$

- dem Auftrieb der unter dem EinfluB der Schwere fallenden Kugel - gesetzt, liefert an Stelle des ,Stokesschen Gesetzes“

die Gleichung

$$
\vec{k}=\frac{2}{9} g R^{2} \frac{s-\sigma}{a}
$$

$$
k=\frac{2}{9} g R^{2} \frac{s-\sigma}{a\left(1+2,4 \frac{R}{\varrho}\right)\left(1+3,3 \frac{R}{h}\right)},
$$

1) Dissertation des Verf. p. 55, Tab. IVb, die zur Berechnung der Reibungskoeffizienten p. 59 verwendet wurde.

2) Diesem Falle entsprechen die Resultate der Tab. IVa; da $R / h$ im Mittel 0,005 ist, ergibt die Theorie, daß in diesem Falle die Kugelgeschwindigkeit im Mittel etwa 1 Proz. kleiner sein muß als in jenem, in Übereinstimmung mit den Experimenten.

3) H. A. Lorentz. l. c. p. 29. 
die für einen oben und unten durch ebene Flächen begrenzten Zylinder vom Radius $\varrho$ und der Höhe $h$ gelten soll, falls die Kugelgeschwindigkeit $a$ im mittleren Drittel der Röhre gemessen wird.

In der Tat werden nun meine experimentell erhaltenen Resultate durch diese Gleichung (8) befriedigend dargestellt: Folgende Tabelle enthält die aus jenen Fallversuchen abgeleiteten Werte des Reibungskoeffizienten (C.G.S.), berechnet nach der unkorrigierten Stokesschen Gleichung $(\bar{k})$ und nach der Gleichung (8) $(k)$; die Dimensionen der verwendeten Kugeln und Röhren und die Werte der beobachteten Geschwindigkeiten finden sich in den zitierten Mitteilungen.

Tabelle.

\begin{tabular}{|c|c|c|c|}
\hline$\frac{R}{\varrho}$ & $\frac{R}{h}$ & $\vec{k}$ & $k$ \\
\hline$\frac{1}{11}$ & $\frac{1}{121}$ & 1681 & 1348 \\
\hline $\begin{array}{c}11,1 \\
1\end{array}$ & 1 & & 1246 \\
\hline 14,7 & $\overline{160}$ & 1600 & 1546 \\
\hline$\frac{1}{10}$ & $\frac{1}{101}$ & 1515 & 1318 \\
\hline 20,0 & 121 & & \\
\hline$\frac{1}{22,2}$ & $\frac{1}{241}$ & 1515 & 1349 \\
\hline$-\frac{1}{26,6}$ & $\frac{1}{160}$ & 1491 & 1340 \\
\hline 1 & 1 & 1490 & 1362 \\
\hline 29,4 & $3 \geq 0$ & & \\
\hline$\frac{1}{35,3}$ & $\frac{1}{1 \simeq 1}$ & 1456 & 1328 \\
\hline$\frac{1}{40,1}$ & $\frac{1}{24 !}$ & 1447 & 1344 \\
\hline$\frac{1}{46,9}$ & $\frac{1}{160}$ & 1438 & 1338 \\
\hline \begin{tabular}{c}
1 \\
\hdashline 3,0
\end{tabular} & $\frac{1}{320}$ & 1424 & 1349 \\
\hline$\frac{1}{70,7}$ & $\frac{1}{241}$ & 1415 & 1348 \\
\hline$\frac{1}{98,5}$ & $\frac{1}{320^{\circ}}$ & 1396 & 1347 \\
\hline
\end{tabular}


Während die aus den Fallversuchen mit verschieden großen Kugeln und in verschieden weiten GefäBen nach der Stokes schen Gleichung berechnete "Reibungskonstante" zwischen 1681 und 1396 variiert, erhält man nach der neuen Gleichung (8) den nahe konstanten Wert

$$
\begin{array}{r}
1343 \\
+\quad 22
\end{array}
$$

und nach der Poiseuilleschen Methode hatte sich für dieselbe Flüssigkeit

ergeben.

$$
\begin{array}{r}
1325 \\
+\quad 15
\end{array}
$$

Cambridge, April 1907.

(Eingegangen 22. April 1907.) 